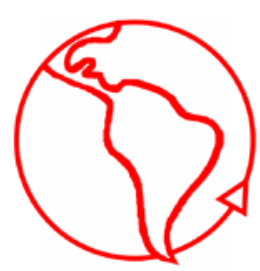

\title{
La observación de la cultura en sistemas organizacionales. Una hipótesis
}

\author{
The observation of culture in organizational systems. A hypothesis
}

Juan Pablo Gonnet

Centro de Investigaciones y Estudios sobre Cultura y Sociedad- Comisión Nacional de Investigaciones Científicas y Técnicas. Universidad Nacional de Córdoba, Argentina

Resumen

En los últimos años la cultura ha dejado de ser una categoría solamente analítica en las ciencias sociales para convertirse en un concepto invocado por distintos actores sociales para describir y observar sus propias prácticas y las de otros. Son las organizaciones, quizás, los actores más destacados en este proceso. Desde la década del ' 80 los teóricos del management y de las ciencias de la administración han señalado que la cultura constituye una dimensión central en todo ámbito organizacional. Esto ha llevado a que la cultura se haya ido convirtiendo en una categoría reflexiva altamente productiva para la autodescripción de sistemas organizacionales. Es el objeto de este trabajo analizar los diversos sentidos involucrados en dichas autodescripciones a partir de dos instancias; por un lado, el discurso del management acerca de las culturas organizacionales y empresariales y por el otro, el análisis comparado de dos situaciones organizacionales (una empresa y una ONG internacional). Intentaremos mostrar cómo la cultura se establece como un modo paradojal de observación y descripción organizacional.

Palabras Clave: Autodescripción; Cultura: Management; Organizaciones; Sentido

\begin{abstract}
In recent years culture has ceased to be just an analytic category in the social sciences to become a concept invoked by social actors to describe and observe their own practices and those of others. Organizations are, perhaps, the most prominent actors in this process. Since the 80 's, management theorists have noted that culture is a central dimension in any organizational environment. In this way, culture has evolved into a highly productive reflexive category for self-description of organizational systems. The aim of this paper is to analyze the meanings involved in such self- descriptions from two perspectives: on the one hand, from the discourse of management about organizational and organizational cultures and on the other hand, from the comparative analysis of two organizational situations (a company and an international NGO). We intend to show how culture is established as a paradoxical mode of observation and organizational description.
\end{abstract}

Keywords: Self- description; Culture; Management; Organizations; Meaning

\section{Introducción}

El objeto de este trabajo es analizar los usos, distinciones y sentidos que son puestos en juego cuando se describe a las organizaciones en términos de cultura. En esta dirección, nos distanciamos de los debates inacabables en el campo de la teoría sociológica, antropológica, organizacional y del management, por lograr una conceptualización adecuada de lo que sería o debería concebirse por cultura organizacional, y nos interesamos más bien, por iniciar una indagación que observe las observaciones 
(observación de segundo orden) ${ }^{1}$ de aquellos que intentan describir a las organizaciones como sistemas comprensibles mediante la semántica cultural. Desde la década del ' 80 la cultura se ha ido constituyendo en una categoría relevante para los investigadores organizacionales. En la actualidad, la cultura es parte del mundo de las organizaciones, llevando a que sus directivos y managers tengan entre una de sus tareas la gestión y el manejo de la cultura organizacional. Aquí, nos interesamos por analizar este proceso a partir del análisis de los discursos del management y de dos casos en la ciudad de Córdoba- Argentina (una empresa y una ONG Internacional), en los cuales miembros de cada una de estas organizaciones utilizan a la cultura como una categoría reflexiva necesaria para la explicación y observación de prácticas y comportamientos organizacionales.

En esta dirección, en primer lugar, retomamos algunas hipótesis propuestas por Luhmann en relación a la cultura como forma de autodescripción social moderna (Luhmann 2007) con el objeto de aplicarla para el caso de las organizaciones y así, distanciarnos de algunas discusiones y modos de indagación que hasta la actualidad se han venido desarrollando en relación a las culturas organizacionales y/o empresariales. En segundo lugar, analizamos las ideas que en el campo del management se han desarrollado en relación con la cultura con el propósito de reconocer los sentidos que atraviesan a la misma. Consideramos que este es un paso necesario para comprender los sentidos que los miembros de las organizaciones (sobre todo managers y directivos) le otorgan a la descripción cultural. En tercer lugar, presentamos dos descripciones de casos con el objeto de reconocer las distinciones y los sentidos que se ponen en juego con la semántica cultural. Finalmente, proponemos la hipótesis de que la cultura organizacional constituye una forma paradójica de observación que favorece la reducción de complejidad en tanto permite reproducir reflexivamente la distinción sistema/entorno; y así, la unidad del sistema social organización.

\section{La cultura como autodescripción}

La idea de cultura organizacional se ha convertido en una categoría exitosa en el campo de los estudios organizacionales. Un indicador de esto lo constituye la vasta literatura sobre la temática y las diversas disciplinas (sociología, antropología, management, teoría de la organización, psicología organizacional, entre otras) que ligadas de una manera u otra al campo de las organizaciones, han buscado incorporarla. La cultura aparece como una dimensión analítica que permite explicar comportamientos organizacionales que eran difíciles de entender en relación a desarrollos teóricos anteriores. Es decir, la cultura se estableció, de una manera u otra, en una "variable" - dependiente, independiente o interpretativa (Smircich 1984)- que otorgaba luz sobre determinados fenómenos organizacionales complejos.

Una de las características generales de esta literatura ha sido una competencia permanente por proponer definiciones cada vez más completas de lo qué es la cultura organizacional. En este sentido, una definición se enfrenta a definiciones anteriores argumentando que hay dimensiones que no han sido consideradas y que deberían serlo. Se presentan situaciones que brindan evidencia de que lo dicho hasta el momento es insuficiente y se pretende superar el estado de la cuestión ${ }^{2}$. Podríamos decir, que la

\footnotetext{
1 “Un observador de segundo orden es un tipo de observador externo, orientado a la observación de observadores y sus respectivas observaciones. Desde su posición no sólo puede observar lo que sus observadores indican y describen -el qué observan, sino también, captar los esquemas de diferencias con que marcan tales observaciones y trazan sus distinciones -el cómo observan" (Arnold 2004: 16).

${ }^{2}$ Esta estrategia es casi constitutiva de la literatura sobre la temática. Véase por ejemplo, Abravanel et al. (1992); Alvesson (2002); Drucker (1996); Gellner y Hirsch (2001); Hallet (2003); Jaime (2002); Luhmann (2010); Ouchi y Wilkins (1985); Rodríguez (2001); Schein (1988); Smircich (1984); Wright (2005). De un modo similar, el concepto de cultura genera este tipo de debates en la antropología (cfr. Kuper 2001).
} 
pregunta constitutiva en este campo de estudios ha sido la de qué es o qué podría ser la cultura organizacional. Este tipo de preguntas, llevan a la producción teórica a una carrera de intentos sin fin por refutar lo dicho y proponer nuevas respuestas que luego serán refutadas y complejizadas. Es decir, la pregunta por el "qué" remite a una esencia de los fenómenos sobre la que es difícil estimular un desarrollo teórico sustentable. En este escrito, nos interesamos por distanciarnos de este proceder y buscamos responder al cómo emerge la cultura como un modo de descripción "adecuado" del mundo de las organizaciones, en otros términos, cuáles son las distinciones que aporta este modo de interpretar a los sistemas organizacionales.

Aquí, nos orientamos por los planteos de Luhmann (2007) en relación a la cultura, quien la entiende como un modo de autodescripción societal. Es ampliamente reconocido que Luhmann no es un teórico social afín al concepto de cultura (Baecker 1997; Martens 2006) fundamentalmente, debido a que considera que el mismo es un concepto sobrevalorado para la comprensión de lo social (Luhmann 1998: 117 y ss.). No obstante, Luhmann no niega el hecho de que la cultura constituya una semántica sumamente relevante desde los inicios de la modernidad para la observación (auto y hetero) de la/s sociedad/es. Para Luhmann (2007) la cultura es una semántica de autodescripción ${ }^{3}$ de la sociedad moderna que emerge en el siglo XVIII como consecuencia de la comparación de modos de vida distintos.

Cultura en el sentido moderno siempre es la cultura reflexionada como cultura, una descripción observada en el sistema. (...) Entonces cultura significa algo así como la forma expresiva anclada en la sociedad, de una representación del mundo, la cual en otras sociedades puede tomar formas diferentes. (Luhmann 2007: 698)

Mediante la semántica de la cultura es posible reconocer la unidad de una sociedad a partir de su diferenciación con otras, de aquí que para Luhmann la cultura siempre signifique pluralidad de culturas. En consecuencia, la cultura, como todo semántica, es un dispositivo de control que permite regular la reproducción del sistema social, distinguiendo lo que posee sentido de lo que no (Baecker 1997: 48). Desde estas premisas, proponemos nuestro análisis de la cultura organizacional. Esto significa que no nos interesamos tanto por una teoría cultural sino que más centralmente problematizamos los usos de la cultura en el campo de las organizaciones contemporáneas.

En este punto debemos aclarar que Luhmann, desde nuestro punto de vista, no es totalmente consistente con esta interpretación societal de la cultura (como semántica) en sus textos ligados a la teoría de la organización (Luhmann 2010, 1997). En éstos, se muestra una pretensión por proponer una definición específica de lo que debería entenderse por cultura organizacional. Luhmann retoma, por ejemplo, la definición propuesta por Rodríguez (2001) para entender a la cultura organizacional como "complejo de premisas de decisión indecidibles" (Luhmann 2010: 281). En esta dirección, tanto Luhmann (2010) como Rodríguez (2005, 2001), se embarcan en una discusión con otras definiciones y concepciones acerca de lo que sería o no conveniente entender como cultura organizacional. En este punto, nuestra perspectiva se separa de la propuesta luhmanniana, debido a que no nos interesamos por discutir una definición "adecuada" de cultura organizacional, sino que pretendemos comprender los sentidos de la categoría cultura aplicada a las organizaciones ${ }^{4}$.

\footnotetext{
${ }^{3}$ Autodescripción es la elaboración en texto de una autobservación (Luhmann 2007: 704).

${ }^{4}$ En este escrito nos centramos, principalmente, en el discurso del management, pero será relevante en futuros trabajos analizar también a las teorías más analíticas en relación a la cultura organizacional. En este caso teorías como las de Luhmann y Rodríguez se convertirían en parte del objeto de observación.
} 
En relación con los estudios sobre cultura organizacional, podríamos asumir que lo que se ha pretendido es formular una teoría que asuma los factores culturales como dimensión interpretativa para la comprensión de fenómenos organizacionales. La cultura (entienda como se entienda) es aquí un nivel de análisis necesario ${ }^{5}$. La estrategia que seguimos aquí, en cambio, consiste en preguntarse por la cultura como forma de autodescripción organizacional e interrogarse por sus usos y sentidos. En los términos de la teoría de sistemas podríamos decir que proponemos un desplazamiento de una observación de primer orden (observación del mundo) a una observación de segundo orden (observación de observadores y de sus distinciones). Esto significa que no pretendemos observar al mundo desde la categoría de la cultura organizacional como concepto analítico, sino que buscamos observar cómo observan aquellos que en los sistemas organizacionales apelan a la idea de cultura ${ }^{6}$. Preguntas relevantes son aquí las siguientes: ¿Qué distinciones se ponen en juego cuando se describe un fenómeno organizacional como cultural?, ¿cuáles son las condiciones de posibilidad de la emergencia de la categoría cultura en el campo de las organizaciones?, ¿frente a qué distinciones se opone la forma cultura en sistemas organizacionales?

\section{La cultura como autodescripción en el campo del management}

Desde fines de la década del ' 70 y comienzos de los ' 80 , la cultura ha sido una categoría que se ha ido desplazando socialmente (Hannerz 2001; Wright 1998; Yúdice 2002). Esto significa que diversos grupos, comunidades y sistemas sociales la han ido incorporando como una dimensión significativa para la descripción y atribución de sentidos en sus prácticas, decisiones, representaciones y acciones. Consideramos que la disciplina del management ha sido uno de estos espacios resignificados por la categoría cultural. En este apartado consideramos pertinente analizar los sentidos de esta penetración de la cultura en el mundo organizacional a partir de los discursos producidos en el campo del management, ya que este es un campo discursivo que, por sus pretensiones, constituye una mediación directa entre el sistema científico y los sistemas organizacionales. No queremos decir que el management sea una descripción de lo que sucede en las organizaciones, pero sí consideramos que el management nutre de sentidos a estos sistemas, los cuales son trasmitidos por medio de profesionales y consultores que ponen en relación la disciplina académica de la administración con las realidades organizacionales (Boltanski \& Chiapello 2007; Reed 1989)7. En términos sistémicos podríamos decir que existe un acoplamiento estructural entre la teoría del management y los sistemas organizacionales ${ }^{8}$.

Siguiendo a Bendix (1966) sostenemos que el management constituye el sistema de ideas (conjunto de saberes) que pretende legitimar la autoridad y el control en sistemas organizacionales $^{9}$. Estas ideas no son azarosas sino que se encuentran ligadas a las

\footnotetext{
${ }^{5}$ Por ejemplo, Alvesson en relación al concepto de cultura organizacional, "The centrality of the culture concept follows from the profound importance of shared meanings for any coordinated action" (Alvesson 2002: 2); "Culture is the setting in which these phenomenon [organizational] become comprehensible and meaningful." (Alvesson 2002: 4).

${ }^{6}$ En relación a este punto es relevante la propuesta de Yúdice (2002), quien concibe que la cultura ya no puede ser utilizada como un dispositivo analítico, sino que debe ser entendida como un recurso al que apelan diversos actores y sistemas para el desarrollo de determinadas estrategias en el mundo social y político.

${ }^{7}$ No obstante, esta relación es un problema que deberá ser referenciado empíricamente en cada caso.

${ }^{8}$ Los acoplamientos estructurales en el marco de la teoría de sistemas sociales autopoiéticos refieren al proceso de auto-sensibilización del sistema a determinadas informaciones del entorno, sin que esto implique una determinación del entorno sobre el sistema. Véase Luhmann (2002: 127 y ss.).

${ }^{9}$ Es notorio remarcar que la mayoría de las escasos acercamientos de la sociología al estudio de las ideas manageriales remita solamente a organizaciones económicas. Por ejemplo, Boltanski y Chiapello (2007), en uno de los estudios más recientes sobre el discurso managerial, consideran que el mismo es una ideología que pretende legitimar al sistema capitalista. No obstante, como mostraremos en este trabajo los discursos manageriales no sólo repercuten en organizaciones del sistema económico. Bendix también limita la comprensión del management al sistema económico:
} 
condiciones sociales en las cuales se producen ${ }^{10}$. En este sentido, el análisis del discurso managerial no es sólo útil como un indicador de las "ideologías que legitiman el control y la dominación" sino que también permite analizar transformaciones sociales, las cuales son indisociables de la producción misma de esos textos ${ }^{11}$. De esta manera, procedemos a analizar, en primera instancia, las condiciones sociales que posibilitan la aparición de la cultura en los discursos del management y en segundo lugar, a las distinciones que se ponen en juego en relación a la cultura en dichos discursos.

Antes de comenzar se vuelve necesario hacer una aclaración. Los textos sobre culturas organizacionales se pueden dividir entre aquellos que poseen un interés más académico y aquellos que explícitamente son prescriptivos en relación al quehacer organizacional. Aquí nos focalizamos en este segundo tipos de textos ya que partimos de la premisa de que son éstos los que mejor se articulan con las necesidades prácticas de la vida cotidiana organizacional. Dichos textos tienen un nivel de teorización y abstracción menor y en general, poseen juicios de valor que se plasman en lineamientos a seguir, consejos, directivas, propuestas prácticas, entre otras. ${ }^{12}$ Sin embargo, lo anterior no implica que asumamos que perspectivas más teóricas y con intereses más académicos no puedan tener un impacto en el mundo de las organizaciones.

En lineamientos generales, la cultura organizacional aparece como respuesta a un conjunto de transformaciones sociales que estaban afectando la gestión organizacional desde fines de la década del ' 70 . La creciente profesionalización, el desarrollo de nuevas tecnologías, las empresas multinacionales y en forma de red, la crítica a los modelos de gestión y control burocrático, entre otros procesos, empiezan a poner en cuestión los saberes clásicos de gestión y administración. Es en este momento en donde la cultura empieza a constituirse como problema y como solución. Schein (1988), por ejemplo, sostiene la tesis de que los principales problemas de dirección empresarial tienen que ver con la cultura y a su vez, argumenta que operando en la esfera de la cultura esos problemas pueden ser solucionados. Peters y Waterman (1982), por su parte, diagnostican que las empresas exitosas son aquellas que poseen culturas fuertes. La literatura managerial, en su mayoría, refiere la cultura empresarial y organizacional a: creencias, valores, sentidos, reglas implícitas, juicios, presunciones y premisas que comparten los miembros de una organización y que influyen decisivamente en las performances organizacionales. Se entiende así que si comprendemos estas dimensiones será posible entender por qué algunas empresas son eficientes y alcanzan la "excelencia" y por qué otras no. Adicionalmente, se asume que es posible que los líderes y los gerentes definan culturas mejor adaptadas a los propósitos de la organización ${ }^{13}$.

Existen dos condiciones sociales que son reiterativamente referenciadas como causas que hacen imprescindible el reconocimiento y el manejo de la cultura organizacional, éstos son: la insuficiencia de los sistemas de control burocráticos; y las transformaciones de las

\footnotetext{
"Todas las ideas sustentadas por o para aquellos que ejercen una autoridad en empresas económicas y que tratan de explicar y de justificar esa autoridad están comprendidas en esa expresión" (Bendix 1966: 2).

10 Perrow (1974), por ejemplo, entiende que el management científico desarrollado por Taylor estaba vinculado a la necesidad de articular autoridad, control y democracia en la sociedad norteamericana de principios del siglo XX.

${ }^{11}$ En este punto, seguimos a Fairclough: "Text are not just effects of linguistic structures and orders of discourse, they are also effects of social structures, and of social practices in all their aspects, so that it becomes difficult to separate out of the factors of shaping texts" (2003: 15).

12 Los textos utilizados fueron seleccionados en relación a las materias de administración y cultura organizacional pertenecientes a la carrera de administración de empresas de la Universidad Empresarial Siglo 21-Córdoba, Argentina. Adicionalmente, incorporamos al corpus algunos textos considerados fundacionales en relación a las culturas empresariales y organizacionales.

${ }^{13}$ Wright $(1998 ; 2005)$ se muestra crítica frente a este hecho, aunque por otra parte, sería incomprensible que el management se interesase por dimensiones que no puedan ser gestionadas.
} 
estructuras sociales de las organizaciones. Ambos cambios implican la necesidad de modificar los supuestos en los cuales se sustenta el control y la autoridad organizacional.

a) En relación a la crisis de los modelos de autoridad formal y de control burocrático, Boltanski y Chiapello (2007), para el caso francés, observan que esto empieza a ser un problema desde la crítica "artista" producida en el mayo francés del ' 68 y que en la década del ' 80 penetra en el discurso empresarial por medio de los profesionales que se integraban al sistema de trabajo. Se estableció así una masa de profesionales que se oponían a formas de gestión burocráticas ya que se consideraba que éstas eliminaban la creatividad, enajenaban a los empleados y limitaban la autonomía. Con respecto a esto, Deal y Kennedy (1985) plantean: "Creemos que la sociedad contemporánea sufre de una incertidumbre generalizada con respecto a los valores, de un relativismo que mina tanto la autoridad de sus dirigentes como su desempeño" (Deal \& Kennedy 1985: 22). En este sentido, para los teóricos del management se define la necesidad de reproducir el control por fuera de las estructuras burocrático-racionales (Peters \& Waterman 1982). "Hay que aprender a dirigir en situaciones donde no se tiene autoridad de mando, en donde no controlas ni te controlan" (Drucker 1996: 12). En este contexto, la cultura aparece como una posible respuesta a esta paradoja. "Puesto que la cultura podría ser un mecanismo para guiar la conducta del empleado, es tan importante como el sistema de remuneración y de evaluación de desempeño" (Hitt et al 2006: 135). Deal \& Kennedy dicen "Creemos que el elemento humano es el recurso más importante con que cuenta una compañía y que la forma de administrar ese recurso no es directamente por medio de informes producidos por un computador, sino mediante el uso de sutiles indicios proporcionados por la cultura" (Deal \& Kennedy 1985: 16) y prosiguen, "Si los empleados saben lo que su compañía representa, es mucho más probable que tomen decisiones que apoyen estas normas; es más probable que se sientan parte de la organización" (Deal \& Kennedy 1985: 22). En esto se puede observar que la cultura se pone como un equivalente funcional al control burocrático. Se considera necesario el paso de Gerentes Racionales a Gerentes Simbólicos (Peters \& Waterman 1982).

b) Otra dimensión social ampliamente reconocida en los textos sobre cultura organizacional tiene que ver con las transformaciones en las estructuras sociales de las organizaciones. La emergencia de organizaciones y empresas multinacionales, los modelos de organización en forma de red, los joint-ventures y el outsourcing, entre otros, aparecen como condiciones estructurales que dan cuenta de la necesidad de organizar mediante sistemas culturales. "Hay cada vez más personas que trabajan en una empresa y que no son empleados de la misma" (Drucker 1996: 28). Ferrel (2004) habla de que las organizaciones dependen cada vez más de sus vinculaciones con proveedores, clientes, sistemas financieros y sistemas de recursos humanos que operan más allá de los límites de la organización. Si a esto se le suma, las transformaciones en el mundo del trabajo descritas por Harvey (2004) y Sennett (2006) acerca de que las expectativas de trabajar toda la vida para una misma organización son cada vez menores, nos encontramos con una transformación de las relaciones sociales para las cuales a la administración no le resulta suficiente remitir a procedimientos y reglamentaciones formales. Se vuelve necesario un sistema de control más abstracto, más general y más "básico". Nuevamente, la cultura como sistema de valores y presunciones se establece como una respuesta posible a estos interrogantes acerca de las condiciones de posibilidad de gestión, control y administración organizacional. Esta cita de Hitt et al (2006) condensa bastante bien lo anterior:

Las organizaciones actuales enfrentan ambientes de negocios más complejos y dinámicos, como quizás en ningún otro momento de la historia. Si una organización intenta crear políticas específicas para todas las situaciones posibles en un ambiente tan dinámico, el resultado sería un manual tan grueso como varias guías telefónicas y, por lo tanto, con muy poco sentido práctico. Además, para cuando tal manual se terminara de imprimir y se iniciara su distribución, es muy 
posible que el ambiente hubiera cambiado lo suficientemente como para que ya fuera obsoleto. $\mathrm{Si}$, por el contrario se dicen a los empleados un conjunto de creencias y valores para que con base a ellos, evalúen situaciones y determinen las acciones a seguir, entonces las organizaciones podrían distribuir un folleto breve y sencillo acerca de los valores de la compañía y dejar que ellos guíen el comportamiento de sus empleados. Entonces, la cultura organizacional, a la cual al principio muchos administradores consideraron como un tema inflado, ahora comienzan a verlo como un asunto estratégico que afecta de manera íntima los cimientos de una compañía. (Hitt et al. 2006: 135)

Más allá de esto, nuestro objeto no es el pensamiento managerial en sí, sino que lo que nos interesa es comprender los sentidos de la categoría cultura utilizadas por la teoría de la administración. Sostenemos que la cultura aparece como una forma de descripción paradojal en el mundo de las organizaciones. Por un lado, la cultura aparece como lo limitante y externo, y por el otro, aparece como lo interno susceptible de ser creado, construido y manejado. Así, la cultura cuando es atribuida al entorno se presenta como estática, fija, indescifrable y hasta irracional; y cuando es atribuida al interior es sinónimo de excelencia, orden, desarrollo y eficiencia.

Como dijimos, la cultura como límite aparece cuando el management reconoce que opera en el marco de "otras culturas", es decir, cuando reconoce que en su entorno hay otros "distintos" con valores y presunciones que limitan las posibilidades de gestión organizacional y por lo tanto, del discurso managerial como discurso con alguna pretensión de validez. Es claro que estos límites no se presentan como inmodificables, si fuera así, el discurso del management no tendría cabida. Es decir, la posibilidad de prescribir formas adecuadas para alcanzar fines específicos caducaría. En este sentido, el límite cultural es, frecuentemente, representado como un límite doblegable. Por ejemplo, Drucker (1995) menciona "Si no se consigue movilizar la herencia cultural específica de un país y un pueblo, es improbable que se promueva el desarrollo social y económico (...) Ahora sabemos que la administración tiene que lograr que los valores, las aspiraciones y las tradiciones de los individuos, la comunidad sirvan a un propósito común" (Drucker 1995: 24). Así, la cultura aparece como lo otro en relación al management y por lo tanto, como lo otro en relación a la productividad y al desarrollo. Podríamos pensarlo como una distinción: Management/Cultura. Hitt et al (2006) plantean que "Los valores sociales son los deseos y condiciones corrientemente compartidos. En términos prácticos para los administradores sociales determinan el grado en el cual los productos y servicios de una organización tienen acceso al mercado" (Hitt et al 2006: 80). La identificación de la cultura en el campo del management remite a una diversidad cultural que el administrador debería enfrentar: "el entendimiento tanto de los fenómenos demográficos como de los valores sociales ayudará a usted, como administrador, a realizar cambios y tomar decisiones que le sirvan para incrementar su eficacia y el desempeño de la organización" (Hitt et al 2006: 80).

No obstante, existe otro sentido casi paradójico con el anterior, en el que se conceptualiza a la cultura como algo interno de la organización, potencialmente manejable y con resultados positivos: "La organización no puede sumergirse en la comunidad ni subordinarse a los fines de ésta. Su cultura tiene que trascender a la comunidad (...) Si la cultura de una organización choca con los valores de la comunidad en la que trabaja, la organización debe prevalecer, de lo contrario no aportará nada a la sociedad" (Drucker 1996: 67-68). Aquí la cultura refiere al sentido procesual de cultivo (cfr. Williams 1994), de desarrollo organizacional. En este movimiento se produce una re-entry (Luhmann 2002) (entrada de la forma en la distinción) de la distinción management/cultura y se distingue entre cultura organizacional/cultura. La cultura organizacional se puede manejar mediante procedimientos tales como la "selección de personal", la "socialización", la "evaluación de desempeño", las "recompensas y la remuneración", la "historia" y los "símbolos". Dicho manejo es productivo y conducente al éxito. "La importancia de la 
cultura corporativa para la gerencia es que influye en la conducta de todos los miembros de la organización, y si se maneja cuidadosamente tendrá un efecto positivo en el éxito de la empresa" (Certo 2000: 393).

Si bien la administración es una disciplina-es decir, un cuerpo organizado de conocimiento, y como tal aplicable por doquier-también es cultura. No es una ciencia desprovista de valores. La administración es una función social y está inserta en la cultura -una sociedad-, una tradición de valores, costumbres y creencias, así como sistemas gubernamentales y políticos. La administración está condicionada por la cultura y es lógico que así sea; pero a su vez, la administración y los gerentes plasman la cultura y la sociedad. (Drucker 1995: XI)

Es decir, la cultura tiene un sentido paradójico en el campo del management. Cuando es atribuida a otros es limitante, crítica, irracional y negativa; cuando es atribuida a la organización, en cambio, es positiva, moldeable, racional, productiva y eficaz. La paradoja radica en que la cultura se hace manejable una vez que es definida como límite. La cultura, en el discurso del management, es aplicada al entorno cuando describe la diversidad de culturas que entran en interacción con la organización y que son inconmensurables y es remitida al management cuando es sinónimo de eficiencia y "excelencia".

\section{La cultura como autodescripción en ámbitos organizacionales}

En el apartado anterior mostramos cómo la cultura se convierte en un concepto significativo en el pensamiento managerial. Ahora bien, esto no necesariamente implica que las organizaciones estén representadas por los discursos del management, sin embargo, en este apartado mostramos cómo dos organizaciones (más precisamente, sus mandos medios) utilizan la cultura como un concepto reflexivo que permite la descripción, el planeamiento, la evaluación y la decisión organizacional. Presentamos, brevemente, a continuación las dos situaciones.

\section{a) Situación 1}

La empresa Factor ${ }^{14}$ cuyo objeto es el incremento de la eficiencia de los procesos administrativos de un organismo gubernamental de la provincia de Córdoba me contacta por medio de la maestría en antropología de la universidad nacional de Córdoba con el objeto de buscar un consultor que sea antropólogo organizacional. El director de Recursos Humanos de Factor envía un mail a la maestría el cual me es reenviado a mi cuenta. El mail es el siguiente:

Como te comenté en nuestra charla telefónica, te cuento un poco lo que necesitamos en la empresa. Los directores esperan una serie de comportamientos por parte del personal y no lo ven reflejado en los actos cotidianos. Notan una diferencia entre lo que los integrantes de la organización valoran y lo que ellos quisieran que valoren. No se perciben como importantes las mismas cosas. Desde la gerencia de RR.HH. hemos identificado que obedece en gran medida a cuestiones del ámbito de la cultura y propusimos contratar un consultor externo para que asesore a la Dirección y a la Gerencia de RR.HH.

Como pautas generales nos fijamos las siguientes que pueden ser modificadas:

1. Generar las condiciones para la gestión de la cultura organizacional.

\footnotetext{
${ }^{14}$ Utilizamos este nombre como un seudónimo de la organización.
} 
- Relevamiento y redefinición de los valores de la empresa.

- Definir la Identidad e imagen que se pretende de la empresa.

- Generar las bases que permitan realizar una gestión de la cultura de FACTOR.

Agradecería que me puedas contactar con alguien con experiencia para esta tarea. Gracias, saludos cordiales.

Acepto la invitación a participar y procedo a tener una entrevista con el director de recursos humanos (Martín ${ }^{15}$ ). Martín es psicólogo con una especialización en psicología organizacional, tiene 35 años, ha hecho cursos y capacitaciones en management, y se encuentra desarrollando su MBA. Lo que procedo a comentar es centralmente esta entrevista. Empiezo la misma preguntándole a Martín, acerca de qué considera que son dimensiones del ámbito de la cultura. Su respuesta remite principalmente a valores organizacionales y sentidos compartidos. El me dice que en Factor no es posible lograr que los empleados entiendan lo que quieren los directivos. Las prácticas cotidianas de los trabajadores no responden a los imperativos y reglamentaciones definidas por la organización. Martín me comenta que la organización ha venido realizando numerosas estrategias para mejorar esto pero sin ningún resultado aparente. Se hacen jornadas al aire libre, reuniones informales, se les da participación a los empleados, se transmite la misión de la organización, pero sin embargo los desacuerdos siguen igual. Son estos hechos los que han llevado a Martín y al equipo de recursos humanos a pensar que el problema es más complejo y profundo, de ahí su deducción de que la inconmensurabilidad de las posturas entre la dirección y los empleados se debe a factores culturales. Martín me comenta que ha escuchado hablar de la temática de las culturas empresariales y el desea constituir y formar una cultura que logre la integración que han obtenido otras empresas (aquí me menciona a empresas bastante reconocidas de la ciudad de Córdoba). Todo esto lo lleva a pensar que lo que hay que hacer es una planificación de la cultura que logre la integración deseada y que evite que toda decisión o reglamentación sea cuestionada.

Es interesante notar que si bien Martín no es una persona que se encuentre plenamente familiarizada con los planteos sobre culturas empresariales reconoce a la categoría cultura como relevante para comprender la realidad de su organización y para plantear soluciones. Esto muestra como los conceptos definidos en las ciencias sociales y en otras disciplinas penetran de distintas maneras al mundo social, incluso sin mediaciones directas con esos saberes. ${ }^{16}$ Más allá de esto, su reconocimiento de la cultura le permite remitirse a la disciplina encargada de su estudio, la antropología.

La cultura es utilizada por Martín como un concepto que le permite hacer comprensible problemáticas organizacionales irresolubles. La cultura emerge como un modo de entender algo que parece incomprensible: ¿por qué más allá de que desde la dirección de recursos humanos se están poniendo en práctica las más diversas estrategias para vincular empleados y dirección, no se logran los resultados esperados? La cultura es el problema, es el reconocimiento de un límite al actuar organizacional. El enfrentamiento entre la gestión y los otros (en este caso, empleados y directivos) hace notar que la situación es cultural. La cultura surge cuando se reconoce que hay modos de hacer, trabajar, pensar, etc. que no son comprensibles para aquel que observa. Por otro parte, nuevamente nos encontramos con la paradoja de que lo inmanejable se vuelve manejable, esto aparece en el planteo de Martín con la pretensión por planificar y gestionar la cultura. Hacer de la cultura una estrategia que permita alcanzar la integración deseada.

\footnotetext{
${ }^{15}$ Nombre ficticio.

${ }^{16}$ Giddens (1993) define a este proceso de amplias implicancias para la teoría social y para la epistemología como doble hermenéutica.
} 


\section{b) Situación 2}

En este caso se trata de una investigación etnográfica que desarrollé en una ONG internacional (opera en toda Latinoamérica) en la ciudad de Córdoba orientada hacia el empoderamiento y desarrollo económico de barrios marginales en grandes ciudades. Dicha organización funciona en Córdoba desde el año 2003 y tiene aproximadamente 150 miembros. Desde sus inicios la sede de esta ciudad ha tenido dificultades para desarrollarse y alcanzar sus objetivos debido a que los empleados locales, aparentemente, no comprenden la lógica organizacional internacional. Fundamentalmente, esta evaluación surge de la comparación de los resultados alcanzados en otros países y los resultados locales. La mencionada situación ha llevado a que los directivos de la organización (tanto a nivel internacional como local) comiencen a desarrollar hipótesis acerca de por qué es diferente la performance de la organización en la ciudad de Córdoba. Es en este punto donde se ha comenzado a hablar de cultura, en su acepción regional-nacional. Se considera que la cultura "cordobesa" es demasiado crítica y que eso lleva a que todos sus miembros cuestionen permanentemente el proyecto organizacional. Se observa que Córdoba es un ambiente crítico, quizás, por la fuerte presencia de su Universidad (lugar del que provienen la mayoría de sus miembros) y sus movimientos políticos y sociales. Se dice que esto lleva a que las acciones de la organización estén frenadas. Un directivo me comenta que esto hace que sea muy difícil transmitir el proyecto organizacional como ya está definido internacionalmente. Así, la cultura local es observada, nuevamente, como el límite que choca con la organización y que la imposibilita en su desarrollo. Para los directivos es incomprensible el hecho de que los miembros "cordobeses" discutan decisiones organizacionales que han probado su eficiencia en otros lugares y que han permitido el desarrollo social.

Frente a este diagnóstico, se define que la cultura de la ONG Internacional es lo suficientemente clara acerca de sus principios, sus valores y su misión y son estas dimensiones las que han permitido que la organización alcance sus fines en tiempo y forma en otros países. Debido a esto, los directivos actuales dicen que su estrategia es reforzar la comunicación de esta cultura organizacional para "socializar" mejor a los miembros actuales y para evitar que entren personas que no sean compatibles con estos principios. Aquí, se reconstruye la cultura regional como algo que puede ser combatido por parte de la cultura organizacional. En este caso la paradoja cultura como posibilidad y cultura como límite se representan en la distinción cultura organizacional/cultura regional (nacional). La cultura organizacional ejemplifica la eficiencia, el desarrollo, el cambio, el bien común, etc., y la cultura regional define la ineficiencia, lo incomprensible y lo improductivo.

\section{Conclusiones: El sentido de la cultura en ámbitos organizacionales}

Para terminar este trabajo nos gustaría explorar el sentido de la cultura como modo de observación en sistemas organizacionales. Entender a la cultura como observación es reconocer que es una indicación que distingue dos lados, uno que es marcado y otro que permanece latente ${ }^{17}$. Luhmann (1998) reconoce que una de las características centrales de los sistemas sociales es su capacidad de procesar sentido para reducir la complejidad de los entornos. En esta dirección, concebimos a la cultura como una forma significativa en los sistemas organizacionales que permite la reducción de complejidad y la absorción de incertidumbre. Siguiendo a Luhmann (1998), concebimos que el sentido se puede descomponer analíticamente en tres dimensiones: la objetiva, la temporal y la social. La objetiva refiere a los temas y a los objetos que se ponen en juego en las comunicaciones; la temporal refiere a la interpretación de la realidad en relación a la diferencia entre el pasado y el futuro; y la social remite a las relaciones entre ego y alter (consenso y disenso)

\footnotetext{
${ }^{17}$ Para la idea de observación como distinción véase Baecker 1999; Luhmann 2007.
} 
(Luhmann 1998: 42 y ss.). Cada una de estas dimensiones es constitutiva del sentido. Para el caso de la cultura en sistemas organizacionales es factible distinguir estas tres dimensiones aunque en las comunicaciones operen en conjunto.

La dimensión objetual involucrada en la cultura se encuentra ligada a la existencia del entorno y de la organización. El entorno es reconocido por medio de la cultura como algo que se impone frente a las decisiones organizacionales. Por otra parte, la organización misma se vuelve tema cuando se definen los alcances de su cultura. Podríamos decir que la cultura hace explícita la diferencia sistema/ entorno y la comunica. Con la semántica de la cultura la organización se reflexiona a sí misma como separada, distanciada y diferenciada del ambiente ${ }^{18}$.

En cuanto a la dimensión temporal, las distinciones mencionadas arriba [organización/cultura] y [cultura organizacional/cultura], remiten a dos momentos distintos. La cultura opera como una distinción en dos instancias. En primer lugar, distingue entre organización (management) y cultura, y en segundo lugar, entre cultura organizacional y cultura. En la primera forma es un límite y en la segunda una posibilidad. Podríamos decir en la primera es un problema y en la segunda, una solución. El antes se liga a la determinación de la cultura y el después a la posibilidad de modificación de la misma. Es interesante ver que la idea temporal de progreso y de racionalización propia de los sistemas organizacionales modernos (Perrow 1974) se reproduce en esta nueva atribución de sentido. Sería interesante explorar si es posible que las organizaciones pudieran desarrollar otros sentidos temporales menos reversibles (¿burocracia?). Es así, que a través de la idea de cultura se puede dar cuenta de que las organizaciones siguen constituyéndose en la solución a los problemas de la sociedad moderna (Weber, Durkheim).

Finalmente, en cuanto a la dimensión social podemos decir que la cultura se plantea preferentemente en el marco del antagonismo entre un ego y un alter. Dichos antagonismos se encuentran más allá de enfrentamientos entre dos grupos sino que más bien se constituyen entre pretensiones significativas que se presentan como contradictorias e incompatibles: cultura y management, organizaciones y cultura, cultura organizacional y cultura nacional. De esta manera, podemos decir, que la cultura aparece cuando en las organizaciones experimentan el antagonismo y la contradicción de sentidos tanto entre sus miembros como con el entorno.

Nos gustaría finalizar planteando que si bien este trabajo estuvo orientado a la comprensión de los sentidos por los cuales las organizaciones se describen en términos de cultura, también estimamos que nuestro diagnóstico puede ser útil para nutrir las reflexiones en torno a los usos y significados de la semántica de la cultura en la sociedad contemporánea. Es posible que algunos de los análisis que presentamos en relación a la cultura y a las organizaciones se encuentren vinculados a la historia de los sentidos de la cultura como autodescripción social. Por ejemplo, Abu-Lughod (1991) considera, para el caso de la antropología, que "Culture operates in anthropoligical discourse to enforce separations that inevitably carry a sense of hierarchy" (Abu- Lughod 1991: 137), es decir, dos elementos (separación y jerarquía) que se encuentran presentes de formas particulares en la idea de cultura organizacional. Desde este lugar, es pensable que el uso de la cultura en el campo de las organizaciones y del management sea comparable a los sentidos que el mismo concepto de cultura acarrea desde sus orígenes.

Reflexiones similares acerca de los usos del concepto de cultura han llevado a dos posicionamientos antagónicos. Por una parte, se encuentran aquellos para los que el

\footnotetext{
${ }^{18}$ En la terminología de la teoría de los sistemas sociales podríamos denominar a este proceso como reflexión, el cual implica que la base de autoreferencia del sistema se desarrolla por la incorporación de la distinción sistema-entorno. Véase Luhmann (1998: 345).
} 
concepto de cultura debe ser abandonado (Abu- Lughod 1991; Wright 1998, 2005) ya que ha dejado de tener un papel interpretativo válido al ser esta una categoría "ideológica". Por otra parte, están aquellos que pretenden redefinir el concepto de cultura con el objeto de subsanar deficiencias de concepciones anteriores (Baecker 1997; Hannerz 2001; Smircich 1984; Williams 1994). La primera alternativa podría decantar en la negación de una categoría "nativa" significativa; la segunda, irremediablemente, es objeto de una permanente deconstrucción. Sin tomar partido por ninguna de estas dos posiciones, consideramos que una tercera alternativa es la que defendimos en este trabajo, es decir, la de la observación de aquellos que observan la cultura. De esta manera, no aceptamos ni rechazamos el concepto de cultura, más bien lo abordamos como un objeto en sí mismo con sus sentidos histórico-sociales específicos. RM

\section{Bibliografía}

Abravanel, H., Allaire, Y., Firsirotu, M., Hobbs, B., Poupart, R. \& Simard, J. (1992). Cultura organizacional. Aspectos teóricos, prácticos y metodológicos. Bogotá: Legis Fondo Editorial.

Abu-Lughod, L. (1991). Writing Against Culture. En R. Fox (Ed.), Recapturing Anthropology: Working in the Present (pp. 137-154). Santa Fe: School of American Research Press.

Alvesson, M. (2002). Understanding Organizational Culture. Londres: Sage Publications.

Arnold, M. (2004). Recursos para la investigación sistémico constructivista. En F. Osorio (Ed.), Ensayos sobre socioautopoiesis y epistemología constructivista (pp.16-25). Santiago de Chile: Ediciones Mad.

Baecker, D. (1999). The Problems of Form. California: Standford University Press.

Baecker, D. (1997). The Meaning of Culture. Thesis Eleven, 51(1), 37-51.

Bendix, R. (1966). Trabajo y autoridad en la industria. Las ideologías de la dirección en el curso de la industrialización. Buenos Aires: Eudeba.

Boltanski, L. \& Chiapello, E. (2007). El nuevo espíritu del capitalismo. Madrid: Editorial Akal.

Certo, S. (2000). Administración moderna. Bogotá: Prentice Hall.

Deal, T. \& Kennedy, A. (1985). Las empresas como sistemas culturales. Ritos y rituales de la vida organizacional. Buenos Aires: Editorial Sudamericana.

Drucker, P. (1996). La administración en una época de grandes cambios. Buenos Aires: Editorial Sudamericana.

Drucker, P. (1995). La gerencia. Buenos Aires: Editorial Ateneo.

Fairclough, N. (2003). Analysing Discourse. Textual Analysis for Social Research. Londres: Routledge.

Ferrel, O. C. (2004). Introducción a los negocios en un mundo cambiante. México D.F.: McGraw Hill.

Gellner, D. \& Hirsch, E. (2001). Inside Organizations. Anthropologists at Work. Oxford: Berg.

Giddens, A. (1993). New Rules of Sociological Method. California: Standford Unviersity Press. (second edition)

Habermas, J. (1998). Problemas de legitimación en el capitalismo tardío. Buenos Aires: Amorrortú.

Hallet, T. (2003). Symbolic Power and Organizational Culture. Sociological Theory, $21(2)$, 128- 149.

Hannerz, U. (2001). Trasnational Connections. Culture, People, Places. New York: Routledge.

Harvey, D. (2004). La condición de la posmodernidad. Buenos Aires: Amorrortu Editores.

Hitt, M., Black, S. \& Porter, L. (2006). Administración. México D.F.: Pearson- Prentice Hall.

Jaime Jr., P. (2002). Um texto múltiplas interpretações: Antropologia hermenutica e cultura organizacional. RAE, 42(4), 72-83.

Kuper, A. (2001). Cultura. La versión de los antropólogos. Barcelona: Paidós. 
Luhmann, N. (1997). Organización y decisión. Autopoiesis, acción y entendimiento comunicativo. Madrid- México D.F.: Anthropos- Universidad Iberoamericana.

Luhmann, N. (1998). Sistemas sociales. Lineamientos para una teoría general. Madrid: Editorial Anthropos- Universidad Iberoamericana.

Luhmann, N. (2002). Introducción a la teoría de los sistemas. Madrid: Editorial AnthroposUniversidad Iberoamericana.

Luhmann, N. (2007). La sociedad de la sociedad. México D.F.: Herder.

Luhmann, N. (2010). Organización y decisión. México D.F.: Herder.

Martens, W. (2006). The Distinctions within Organizations: Luhmann from a Cultural Perspective. Organizations, $13(2)$, 83- 108.

Ouchi, W. \& Wilkins, A. (1985). Organizational Culture. Annual Review of Sociology, 11 (3), 457- 483.

Perrow, C. (1974). Complex Organizations. A Critical Essay. Illinois: Scott, Foresman and company.

Peters, T. \& Waterman, R. (1982). In Search of Excellence. Lessons from America's Best Run Companies. New York: Harper and Row Publishers.

Reed, M. (1989). The Sociology of Management. Lancaster: Harvester Wheatsheaf.

Rodríguez, D. (2005). Diagnóstico organizacional. Santiago de Chile: Alfaomega.

Rodríguez, D. (2001). Gestión organizacional. Elementos para su estudio. Santiago de Chile: Ediciones Universidad Católica de Chile.

Schein, E. (1988). La cultura empresarial y el liderazgo. Una visión dinámica. Barcelona: Plaza y Janes Editores.

Sennett, R. (2006). La corrosión del carácter. Las consecuencias personales del trabajo en el nuevo capitalismo. Barcelona: Editorial Anagrama.

Smircich, L. (1984). Organizational Culture. Administrative Science Quarterly, 28(3), 339358.

Williams, R. (1994). Sociología de la cultura. Barcelona: Paidós.

Wright, S. (1998). The Politicization of Culture. Anthropology Today, 14(1), 7- 15.

Wright, S. (2005). Anthropology of Organizations. Londres: Routledge.

Yúdice, G. (2002). El recurso de la Cultura. Barcelona: Gedisa.

\section{Sobre el autor}

Juan Pablo Gonnet es investigador del Centro de Investigaciones y Estudios sobre Cultura y Sociedad-Comisión Nacional de Investigaciones Científicas y Técnicas CIECS- CONICET, de la Universidad Nacional de Córdoba, Argentina. Magíster en Antropología por la Universidad Nacional de Córdoba, Argentina. Entre sus áreas de especialización se encuentran: sociología de la organización, sociología de la sociología y teoría sociológica. Entre sus últimas publicaciones se destacan: Valores compartidos y conflicto (Revista Trabajo y Sociedad, 2013), ¿Cómo es posible el orden organizacional? (Revista Astrolabio, 2012) y Las distintas conceptualizaciones del riesgo en la sociología (Revista Akrópolis, 2011).

ciecs@ciecs-conicet.gob.ar

\section{Contacto}

Universidad Nacional de Córdoba, Argentina.

Av. Gral. Paz 154, 2 do. Piso.

Tel: 0351- 4341124.

Recibido: Septiembre 2012

Aceptado: Octubre 2012 